\title{
Maize haplotype with a helitron-amplified cytidine deaminase gene
} copy

\author{
Jian-Hong $\mathrm{Xu}$ and Joachim Messing*
}

Address: Waksman Institute of Microbiology, Rutgers University, Piscataway, NJ 08854, USA

Email: Jian-Hong Xu - jianhong@waksman.rutgers.edu; Joachim Messing* - messing@waksman.rutgers.edu

* Corresponding author

Published: 09 November 2006

BMC Genetics 2006, 7:52 doi:10.1 186/147|-2156-7-52
Received: 22 August 2006

Accepted: 09 November 2006

This article is available from: http://www.biomedcentral.com/I47/-2/56/7/52

(c) $2006 \mathrm{Xu}$ and Messing; licensee BioMed Central Ltd.

This is an Open Access article distributed under the terms of the Creative Commons Attribution License (http://creativecommons.org/licenses/by/2.0), which permits unrestricted use, distribution, and reproduction in any medium, provided the original work is properly cited.

\begin{abstract}
Background: Genetic maps are based on recombination of orthologous gene sequences between different strains of the same species. Therefore, it was unexpected to find extensive noncollinearity of genes between different inbred strains of maize. Interestingly, disruption of gene collinearity can be caused among others by a rolling circle-type copy and paste mechanism facilitated by Helitrons. However, understanding the role of this type of gene amplification has been hampered by the lack of finding intact gene sequences within Helitrons.

Results: By aligning two haplotypes of the $\mathrm{z} / \mathrm{Cl}$ locus of maize we found a Helitron that contains two genes, one encoding a putative cytidine deaminase and one a hypothetical protein with part of a $40 \mathrm{~S}$ ribosomal protein. The cytidine deaminase gene, called $Z m C D A 3$, has been copied from the ZmCDA I gene on maize chromosome 7 about 4.5 million years ago (mya) after maize was formed by whole-genome duplication from two progenitors. Inbred lines contain gene copies of both progenitors, the $Z m C D A I$ and $Z m C D A 2$ genes. Both genes diverged when the progenitors of maize split and are derived from the same progenitor as the rice OsCDAl gene. The $Z m C D A I$ and $Z m C D A 2$ genes are both transcribed in leaf and seed tissue, but transcripts of the paralogous $Z m C D A 3$ gene have not been found yet. Based on their protein structure the maize CDA genes encode a nucleoside deaminase that is found in bacterial systems and is distinct from the mammalian RNA and/or DNA modifying enzymes.

Conclusion: The conservation of a paralogous gene sequence encoding a cytidine deaminase gene over 4.5 million years suggests that Helitrons could add functional gene sequences to new chromosomal positions and thereby create new haplotypes. However, the function of such paralogous gene copies cannot be essential because they are not present in all maize strains. However, it is interesting to note that maize hybrids can outperform their inbred parents. Therefore, certain haplotypes may function only in combination with other haplotypes or under specialized environmental conditions.
\end{abstract}

\section{Background}

Gene sequences of closely related plant species as heterologous markers of genetic maps made it possible to align chromosomal regions of these species according to the order of genes conserved through speciation, also called orthologs [1]. Although early genomic sequence analysis of small orthologous regions of closely related grass species already indicated that gene order is interrupted by 
genes that have moved around the genome [2-4], the extent of gene mobility during or after speciation could not be appreciated until the comparisons of larger chromosomal intervals from maize and rice [5]. While the extent of non-collinearity among closely related plant species was surprising in view of the comparison of closely related mammalian species, it was always assumed that within the same species gene order was preserved. The conserved gene order within the same species has been the mainstay of genetic maps because meiotic recombination occurs primarily within genes $[6,7]$ or unique, but complete homologous sequences [8].

However, several comparative sequence analyses among maize inbred lines have now shown that haplotype variability within the same species did not only differ by transposable element insertions but also by the presence of non-allelic gene copies [9-11]. Although retrotranspositions account for major dissimilarities between aligned regions, more than one-third of the predicted genes (27/ 72) are absent in one of the inbreds (B73 and Mo17) at the loci 9002, 9008 and 9009 [11]. However, in all these cases the inserted genes are incomplete and represent gene fragments except at the $z 1 C 1$ locus in BSSS53 [10]. At the $z 1 \mathrm{C} 1$ locus, $\mathrm{B} 73$ has lost four gene copies of the zein gene family that are present in BSSS53, while BSSS53 has gained three additional copies compared to B73. One of the lost genes and two of the added genes are functional and expressed. Moreover, the two new genes have changed in their transcriptional regulation [12]. While the change in copy number of tandemly arranged genes is not unexpected, there were also insertions of unlinked genes. B73 had the insertion of a RAV-like B3-domain DNAbinding protein putative gene, conserved on rice chromosome 3 (1e-143) and BSSS53 has the insertion of two genes, a putative cytidine deaminase gene and a hypothetical gene encoding part of a $40 \mathrm{~S}$ ribosomal protein [10]. While the RAV-like gene in B73 is a gene fragment linked to a transposable element (Xu and Messing, unpublished data), reminiscent of the Pack-MULEs [13], the two genes in BSSS53 appear to have complete open reading frames.

At this point, it is unclear how widespread non-collinearity is among cultivars of other species. Comparison of orthologous regions of two subspecies of rice, O. sativa japonica and indica, in one study did not show insertion or deletions of genes or gene fragments [14]. However, comparison of rice chromosome 4 in those two subspecies shows examples of insertion and deletion of genes [15]. Comparison of orthologous regions around the Rph 7 locus of two barley cultivars also differed by at least one gene that could encode a helicase [16]. Therefore, it appears that maize inbred lines stand out in the degree of intra-species gene non-collinearity among other species studied so far. The underlying question is what is the selective advantage of gene mobility and what is the mechanism that allows genes to move from one location to another?

One could hypothesize that moving genes into a different chromosomal context could provide novel regulation of an existing gene function or produce novel gene function [17]. If it results from a "copy and paste" rather than a "cut and paste" mechanism, it also could provide redundancy of a critical function or could contribute to one of many quantitative trait loci (QTLs). Recently, it was shown that the imprinted gene Peg10, important for early mouse development, is inserted into a retrotransposon [18]. Similarly, a gene expressed during seed development [19], the maize Fie2 gene, is also inserted into a retrotransposon [20]. Since in both cases the genes contain introns and are single copy genes, they could not have moved there by retrotransposition. Both of these cases are likely derived from a "cut and paste" mechanism, which is different to the examples of non-collinear genes described in this study. Sequence alignments of allelic and non-allelic gene copies have shown that these insertions except for the z1C1 genes were due to a novel type of DNA transposition that is based on a "copy and paste" mechanism [21,22]. Because this mechanism requires a helicase to initiate a rolling circle-type of replication, the transposed unit is referred to as Helitron.

The Helitrons were first identified by computational analysis in Caenorhabditis elegans, Arabidopsis thaliana and Oryza sativa [23]. Helitrons have conservative 5'-TC and CTRR-3' and do not have terminal inverted repeats and target site duplications. Instead, they contain 16- to 20-bp hairpins 10-12 nucleotides upstream from the 3 '-end and transpose precisely between the 5 '-A and $3^{\prime}-\mathrm{T}$, with no modifications of the AT target sites. Autonomous Helitrons encode a 5'-to-3' DNA helicase and nuclease/ligase similar to those encoded by known rolling-circle replicons. Together with their multiple diverged non-autonomous descendants, Helitrons constitute $2 \%$ of both the Caenorhabditis elegans and Arabidopsis thaliana genomes. In maize, non-autonomous Helitron insertions carrying gene fragments are responsible for loss-of-function mutations in the shrunken 2 gene and the barren stalk 1 gene $[24,25]$. Examination of donor and target sites between two inbred lines of maize have then illustrated that at the donor site a portion of an intact gene is copied and inserted into an unlinked genomic location. While the donor site is conserved with allelic versions of a regular gene in different maize inbreds, the target site creates a disruption in gene order and a non-allelic gene copy [22]. In all cases so far the transposed genes by Helitrons are gene fragments or pseudogenes [21]. 
Here, we report an example where a Helitron contains an intact gene that encodes a putative cytidine deaminase (CDA). Cytidine deaminases are a superfamily, which includes Cytidine deaminase, nucleoside deaminase, deoxycytidylate deaminase and riboflavin deaminase. They are enzymes that de-aminate cytidine to uridine and play an important role in a variety of pathways from bacteria to man. Ancestral members of this superfamily were only able to de-aminate cytidine of mononucleotides or nucleosides. Recently, a family of enzymes, the APOBEC family of mRNA editing enzymes, has been discovered that have the ability to de-aminate cytidines not only of RNA $[26,27]$, but also of DNA $[28,29]$. However, the role of CDAs in plants is still poorly understood. A CDAencoding gene from Arabidopsis has been characterized as a housekeeping gene [30], but whether there are CDA genes with other functions and in other plant species still needs to be explored. We, therefore, took advantage of the variability of maize haplotypes to examine not only the contribution of Helitron action to CDAs in maize inbred lines but also the organization of CDA-encoding genes in the maize genome, their expression, and their putative function by their phylogenetic relationship to other CDAs.

\section{Results and Discussion \\ A new Helitron at the $\mathrm{zICI}+\mathrm{BSSS} 53$ locus}

To unravel the mechanisms by which haplotypes of maize acquire the insertion of new sequences, we took advantage of the recently sequenced regions of the z1C1 locus on chromosome 4 from two inbred lines. The chromo- somal region from BSSS53 is a contiguous sequence of 435,076 bp [GenBank:AF528565, AF090447] and from B73 263,630 bp [GenBank:AC144717, AC144718]. When these two sequences are aligned, the two intervals differ by about $100 \mathrm{~kb}$ in size. Expansion of the BSSS53 region is in part due to additional retrotransposition events and in part due to gene insertions [10]. Insertions can be classified by their target site sequences. We therefore aligned the B73 sequences surrounding the insertion in the BSSS53 sequence to determine the end points of the insertion and the sequences at the ends of the insertions.

Transposable elements were easily recognized by their target site duplications, but the amplification of zein genes in BSSS53 did not show any specific target site sequences. However, the result was different for the presence and absence of putative genes that could encode a cytidine deaminase and a partial 40S-ribosomal protein. This insertion in BSSS53 is 5,233 bp long and exhibits the hallmarks of a Helitron $[23,24]$. Based on sequence alignment of the two inbreds the insertion occurred between the AT target sequence. It lacks the target site duplication seen for transposable elements, but it has the typical 5'-TCT and 3'CTAG ends. However, coding information for the helicase is lacking and replaced by one putative intact gene encoding a cytidine deaminase (CDA) and a hypothetical gene encoding a part of an unknown and part of a 40S-ribosomal protein (Fig. 1A).

To further characterize this insertion in BSSS53, we aligned the end sequences with the end sequences of other

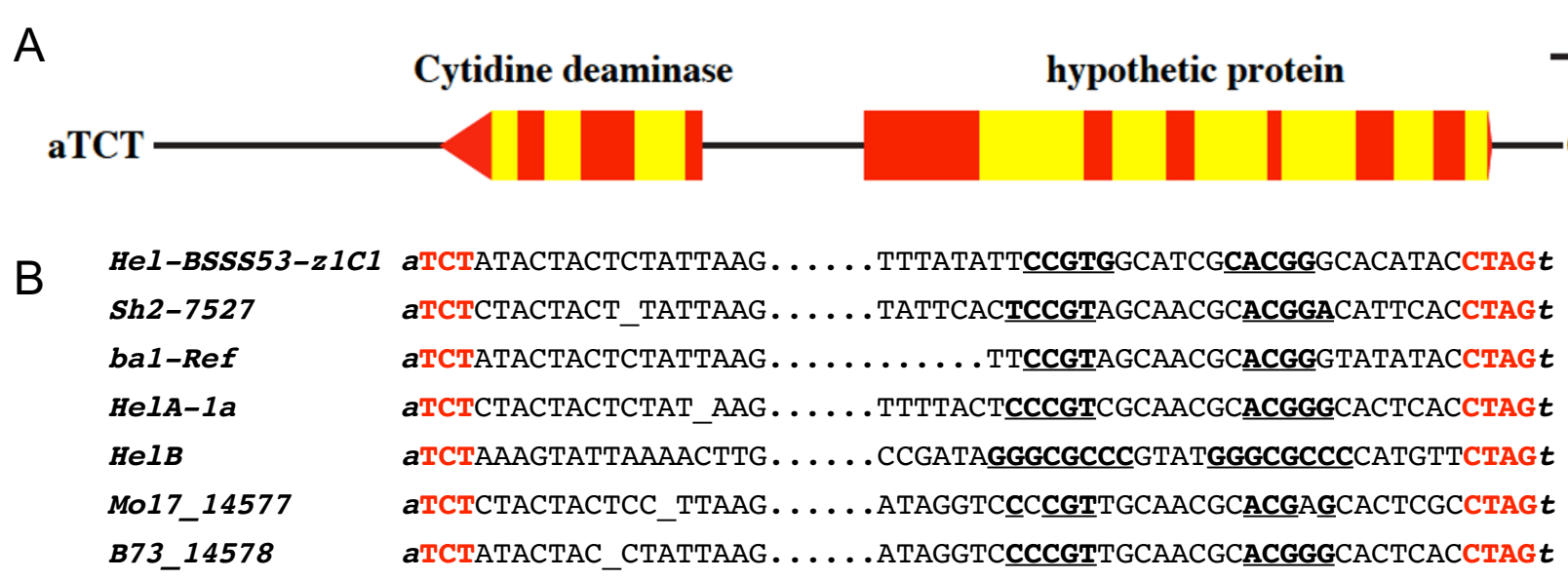

\section{Figure I}

Hel-BSSS53-z I CI on maize chromosme 4S. A) A physical map of Hel-BSSS53-z I CI is presented. Putative genes are shown as pentagons pointing in the direction of transcription. Exons are shown in red and introns in yellow. Conserved nucleotides at the 5'-TCT and 3'-CTAG termini and 5'-a, 3'-t target sites are highlighted at the ends. B) Termini of the maize Helitrons HelBSSS53-z ICI from BSSS53 4S [10], HelA-I $a$ and HelB from McC 9S [22], Mol 7_ 4577 from Mol7 9L, B73_I4578 from B73 6S [II], the Helitron insertions in mutants sh2-7527 [24] and ba I-Ref [25, 49]. Helitron sequences are in uppercase letters and the invariant host nucleotides, where the Helitrons inserted, are in italic lowercase letters. Conserved nucleotides at the 5' and 3' termini are in red bold uppercase letters and inverted repeats at the 3 ' termini are underlined. 
known Helitrons in maize. In addition to the $5^{\prime}$ and 3 ' end sequences we also can find a 16-bp region capable of forming a hairpin structure that is conserved among the maize Helitrons (Fig. 1B). We named this insertion Hel-

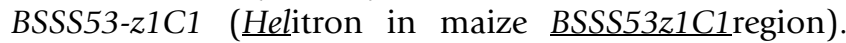
While the sequence features of the end sequences of the BSSS53 insertion are conserved with the other Helitrons, it differs from them by having putative genes with complete coding potential.

\section{Organization of cytidine deaminase (CDA) genes in maize inbred lines}

Because the hallmark of Helitrons is a "copy and paste" mechanism, one would expect that both B73 and BSSS53 would share a common donor site for the sequences contained in $\mathrm{Hel}$-BSSS53-z1C1. We decided to select the CDA gene sequence rather than the sequence for the hypothetical gene encoding part of the $40 \mathrm{~S}$ ribosomal protein for such an analysis because of the value of an intact gene with a known function for tracing Helitron action. We first cloned a CDA cDNA clone based on the CDA gene sequences in the Helitron by reverse transcription of leaf mRNA (Methods) and used it in Southern blot analysis of these two inbreds along with other common inbreds A188, B37, Mo17, and W64A (Fig. 2). As restriction enzymes are chosen that do not cleave within the maize CDA genomic sequence, we can find evidence for a small multigene family as it has been observed in Arabidopsis as well [30]. Therefore, screening of a putative donor site of the CDA gene of the Helitron required a high stringency to filter out CDA genes that are distantly related and possibly have different specificities.

Higher stringency was afforded by BLAST search of BAC end sequences (BESs) with the CDA gene of the Helitron [31]. BESs are anchored to the genetic map through the fingerprinted B73 physical map [32,33]. A single BAC contig \#299 on chromosome 7 was identified and a clone,

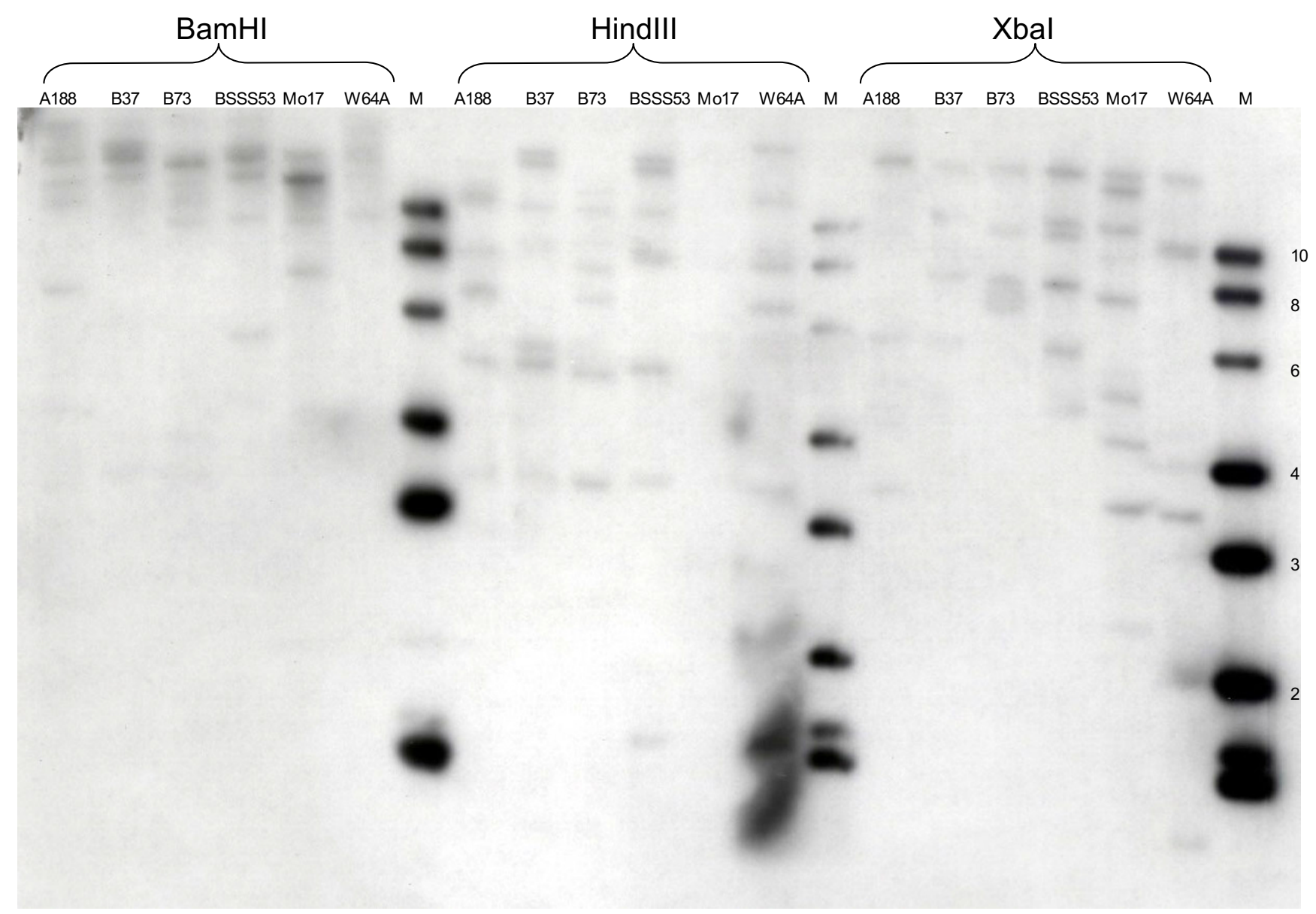

Figure 2

Analysis of genomic CDA-related sequences in maize inbred lines. Genomic DNA from various inbred lines have been digested with BamHI, HindIII and Xbal and subjected to Southern blot analysis as described under Methods. The names of each inbred line are marked above the lanes. 
b0390I10, was selected for sequencing that contained the CDA sequence in the center of the clone. Indeed, b0390I10 contained a putative gene encoding a complete CDA [GenBank: EF106973]. If this putative gene on chromosome 7 is the original maize CDA donor gene, one would expect it to be present in all inbred lines. We therefore constructed a library of genomic sequences from 15 common inbreds that have been amplified with a set of primers of CDA sequences conserved between the chromosome 7 and 4 locations. From each inbred 24 clones were sequenced to quantify the number of genes present in each genome by sequence cluster analysis and insert size [34]. Based on this analysis we obtain four different CDA gene copies, which represent a subset of those detected by Southern blot analysis. Only two genes were present in all inbred lines (Table 1) including the one of clone b0390I10 on maize chromosome 7. We named this CDA gene $Z m C D A 1$. It is not unexpected to find two copies in all inbred lines because maize formed by the hybridization of two closely related progenitors 4.8 mya [37]. We therefore named the second gene copy that is present in all inbred lines $Z m C D A 2$. While B73 seems to have only these two copies of the CDA gene, other inbred lines like BSSS53, Mo17, A654, B37 and CO159 have an additional copy that appears to be in common. Based on sequence cluster analysis this copy corresponds to the chromosome 4 location of BSSS53. We named this copy $\mathrm{ZmCDA3}$, which is the one contained in the Hel-BSSS53z1C1 Helitron. In addition, we can find at least one more copy that is also present in A188, BSSS53, Tx303, CO159, CM37, W64A, Mo17, A632 and A636. However, this copy appears to have a 39-bp deletion in the last exon. Additional truncated copies could be present in some of the inbreds as well. Therefore, the only intact genes of this subfamily of CDA genes in maize are ZmCDA1, ZmCDA2, and $Z m C D A 3$.

\section{Relationship of CDA genes in maize}

We used the $Z m C D A 1$ sequence for a BLAST search [31] of the rice genome, Arabidopsis genome, and the sorghum EST collections. We could identify one copy [GenBank: AAR88587] on rice chromosome 3, which we named OsCDA1. We also identified one related sequence on Arabidopsis chromosome 5, which we named AtCDA10, and one related sequence from sorghum EST data [GenBank: CN133157.1, gi:45963787], which we named SbCDA1. The three maize genes and the related sequences from rice, Arabidopsis, and sorghum were translated and their amino acid sequences compared (Fig. 3). Because rice chromosome 3 contains orthologous regions to maize chromosome 7 [35], it is likely that the rice OsCDA1 gene and the maize $Z m C D A 1$ gene are orthologous and derived from a common ancestral chromosome. If that is the case, then $Z m C D A 1$ and $Z m C D A 2$ should be closer to rice than $Z m C D A 3$. Indeed, based on amino acid homology $Z m C D A 1$ and $Z m C D A 2$ are closer to rice than $Z m C D A 3$ (not shown). Because $Z m C D A 3$ appears to be closer to $Z m C D A 1$ than to $Z m C D A 2$, the Helitron has probably copied $Z m C D A 1$ rather than $Z m C D A 2$. To further analyze the relationship of $Z m C D A 1$ and $Z m C D A 3$, their genomic sequences were aligned as well. From this comparison, it appears that the deletion of the amino-terminal amino acids is due to a deletion of a small part of the first exon and a larger part of the first intronic sequence of $Z m C D A 1$ (Fig. 4). Interestingly, a putative de-amination of the seventh codon restored an ATG start codon and a complete open reading frame for the $Z m C D A 3$ gene. Otherwise, the aligned sequences including the introns are highly conserved (94.6\%). From this analysis, it appears that the progenitors of rice and maize had a common CDA gene that got duplicated during the speciation of maize. The two copies are therefore orthologous gene copies. Subsequently, a Helitron initiated the replication of one of the copies, the one on maize chromosome 7 . During this process the 5 ' region got truncated and a new start-codon generated. This third copy got integrated into a new chromosomal location on maize chromosome 4 along with other sequences including those that are part of a $40 \mathrm{~S}$ ribosomal protein gene. The third CDA gene copy is paralogous and arose after speciation and therefore contributes to a unique haplotype.

\section{Distance analysis of CDA genes}

If the chronology of events is correct, we can determine the timing of these events based on the nucleotide substitutions of each gene pair. As molecular studies have suggested an upper bound of 50 million years ago (mya) for the origin of rice [36], we make the assumption that the

Table I: Summary of ZmCDA patterns in 15 maize inbred lines*.

\begin{tabular}{lcccccccccccccccccccc}
\hline & A632 & A636 & A654 & B37 & Mol7 & W22 & W23 & W64A & CM37 & R232 & COI59 & Tx303 & Al88 & B73 & BSSS53 \\
\hline$Z m C D A 1$ & + & + & + & + & + & + & + & + & + & + & & + & + & + & + & + \\
$Z m C D A 2$ & + & + & + & + & + & + & + & + & + & + & + & + & + & + & + \\
$Z m C D A 3$ & - & - & + & + & + & - & - & - & - & - & + & - & - & - & + \\
Truncated & + & + & - & - & + & - & - & + & + & - & + & + & + & - & + \\
\hline
\end{tabular}

* Sequences of amplified genomic DNA from each inbred with primer pairs ZmCDAF3/ZmCDAR3 clustered as consensus sequences; presence of consensus sequence $(+)$, absence of consensus sequence $(-)$. 


\begin{tabular}{|c|c|c|c|}
\hline$Z m C D A 1$ & 11 & MDEAQVVESKDGTISVASAFAAHQEAVQDRDHKFLTKAVEEAYRGVDCGDGGPFGAVVVC & 60 \\
\hline$Z m C D A 2$ & 11 & MDEAQVVESKDGTISVASAFAGFQEAVQDKDHKFLTKAVEEAYRGVDCGDGGPFGAVVVR & 60 \\
\hline$Z m C D A 3$ & $1-$ & ------MESKDGTISFASAFAAHQEAVQDRDHKFLTKAVEEAYRGVDCGDEGPFGAVVVR & 54 \\
\hline$S b C D A 1$ & 11 & MDEAQVVESKDGTISVASAFAGHQEAVQDRDHKFLTKAVEEAYRGVDNGDGGPFGAVVVR & 60 \\
\hline OSCDA 1 & 11 & MEEAQVVESKDGTISVASAFAGHQEAVQDRDHKFLSKAVEEAYQGVDCGHGGPFGAVVVR & 60 \\
\hline AtCDA 10 & 11 & $\begin{array}{l}\text { MEEAKV-EAKDGTISVASAFSGHQQAVHDSDHKFLTQAVEEAYKGVDCGDGGPFGAVIVH } \\
\text {. } \ldots \ldots * * * * * * * . * * * * \ldots * * * * * * * * * * * \ldots * * * * * * * * * . * \ldots * * * * * . * .\end{array}$ & 59 \\
\hline$Z m C D A 1$ & 611 & NDEVVVSCHNMVLKHTDPTAHAEVTAIREACKKLGKIELSDCEIYASCEPCPMCFGAVHL & 120 \\
\hline$Z m C D A 2$ & 611 & NDEVVVSCHNMVLKHTDPTAHAEVTAIREACKKLGKIELSDCEIYASCEPCPMCFGAVHL & 120 \\
\hline$Z m C D A 3$ & 551 & NDEVVVSCHNMVLKHTDPIAHAEVTAIREACKKLGKIELSDCEIYASCEPCPMCFGALHL & 114 \\
\hline$S b C D A 1$ & 611 & NDEVVVSCHNMVLKHTDPTAHAEVTAIREACKKLGKIELSDCEIYASCEPCPMCFGAVHL & 120 \\
\hline OSCDA 1 & 611 & NDEIVVSCHNMVLDYTDPTAHAEVTAIREACKKLGKIELSDCEMYASCEPCPMCFGAVHL & 120 \\
\hline AtCDA 10 & 601 & 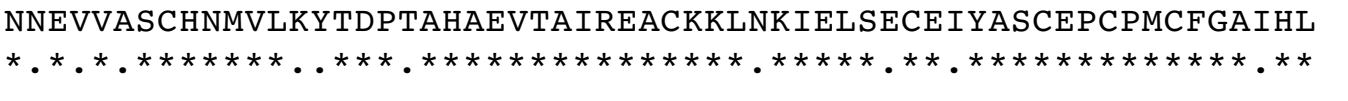 & 119 \\
\hline$Z m C D A 1$ & 121 & SRIKRLVYGAKAEAAIAIGFDDF IADALRGTGFYQKANMEIKKADGNGALIAEQVFEKTK & 180 \\
\hline$Z m C D A 2$ & 121 & SRIKRLVYGAKAEAAIAIGFDDF IADALRGTGFYQKANLEIKKADGNGALIAEQVFEKTK & 180 \\
\hline$Z m C D A 3$ & 115 & SRIKRLVYGAKAEAAIAIGFDDF IADALRGTGFYQKANME I KKADGNGAL IAEQVFEKTK & 174 \\
\hline$S b C D A 1$ & 121 & SRIKRLVYGAKAEAAIAIGFDDF IADALRGTGFYQKANME I KKADGNGAL IAEQVFEKTK & 180 \\
\hline OSCDA 1 & 121 & SRIKRLVYGAKAEAAIAIGFDDF IADALRGTAYYQKANLEIRRADGNGALIAEQVFENTK & 180 \\
\hline AtCDA 10 & 120 & $\begin{array}{l}\text { SRLKRLVYGAKAEAAIAIGFDDFIADALRGTGVYQKSSLEIKKADGNGAAIAEQVFQNTK } \\
* * . * * * * * * * * * * * * * * * * * * * * * * * * * * * * \ldots * * * \ldots * * \ldots * * * * * * . * * * * * * \ldots * *\end{array}$ & 179 \\
\hline$Z m C D A 1$ & 181 & EKFQMY & 186 \\
\hline$Z m C D A 2$ & 181 & EKFQMY & 186 \\
\hline$Z m C D A 3$ & 175 & EKFQMY & 180 \\
\hline$S b C D A 1$ & 181 & EKFRMY & 186 \\
\hline$O S C D A 1$ & 181 & EKFRMY & 186 \\
\hline$A t C D A 10$ & 180 & EKFRLY & 185 \\
\hline
\end{tabular}

\section{Figure 3}

Alignment of amino acid sequences of related CDAs from maize, sorghum, rice, and Arabidopsis. The coding sequences of CDAs from maize and other organisms have been translated into single letter amino acid code and aligned using the Laser gene program as described under Methods. Gene names are marked at the beginning of the sequence. Conserved amino acid positions are highlighted by stars $(*)$.

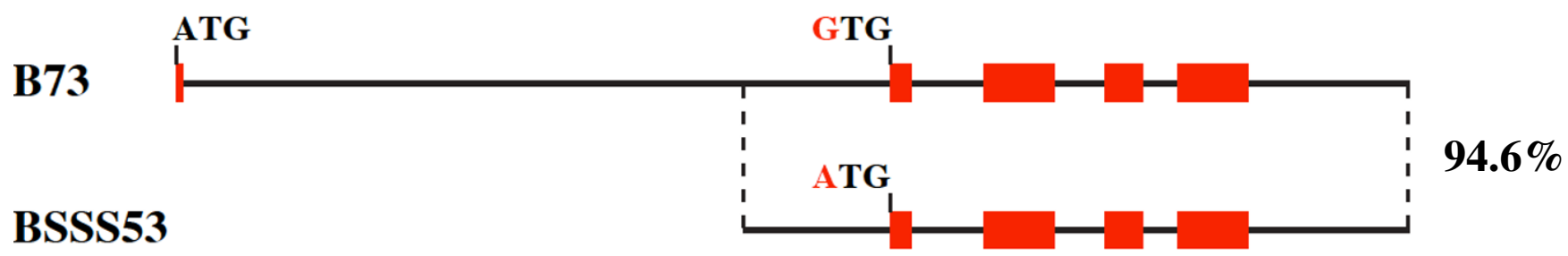

Figure 4

Alignment of the ZmCDAI and ZmCDA3 genes. The coding sequences of the ZmCDAI and ZmCDA3 genes from B73 and BSSS53 are depicted graphically as bars to illustrate their common sequences. The start codons are highlighted. Exons are shown in red block, nucleotide change from $G$ to $A$ is shown in red letters. 
progenitors of maize and sorghum separated from the progenitor of rice 50 mya. Using this divergence date, we estimated the average nucleotide substitution rate for the maize CDA genes and determined the distance between the $Z m C D A 1$ and $Z m C D A 2$ genes. They appear to have diverged about 14.5 mya, which is higher than the 11.9 mya observed previously [37]. However, the previous study also found that synonymous distances between different orthologs of maize and sorghum could vary 3.2fold. If we calculate the distance of maize and rice CDA orthologs, they would have diverged 12.8 mya. Therefore, the maize CDA orthologs are within the same range of nucleotide substitution rates as previously studied ones and support the allotetraploid model of the origin of the maize genome.

Because the $Z m C D A 3$ gene also has an intact coding sequence it provides us for the first time the opportunity to determine when Helitron movement occurred. We make the assumption that conservation of an intact gene has the same substitution rate regardless whether is expressed constitutively or under specialized conditions. Based on the synonymous nucleotide substitutions of the $\mathrm{ZmCDA3}$ gene relative to the donor gene $\mathrm{ZmCDA1} \mathrm{Heli-}$ tron movement occurred about 4.5 mya (Fig. 5), which is just after the hybridization of the progenitors of maize, estimated to be 4.8 mya [37]. Interestingly, it has been shown that the majority of retrotranspositions also occurred after the hybridization of the two progenitors in maize as early as 4.6 mya but with $74 \%$ less than one mya [20]. Similarly, paralogous copies of zein genes arose 4.3, 2 , and 0.5 mya [12]. Therefore, within the same time frame different mechanisms have triggered amplification and integration of sequences into the maize chromosomes including Helitrons.

\section{Cytidine deaminase gene expression in maize inbred lines}

To investigate whether this subfamily of CDA genes is expressed in maize, we tested tissues for these CDA transcripts. We sampled total mRNA from leaf tissues of adult plants from six typical inbred lines A188, B37, B73, BSSS53, W64A and Mo17 and from 20-days old immature endosperm of BSSS53 and B73. The mRNA was amplified by RT-PCR, cloned and analyzed by sequence analysis [GenBank: EF105328-EF105335]. Alignment of RT-PCR products shows that the mRNA is derived from the $Z m C D A 1$ and the $Z m C D A 2$ genes (Fig. 6). Having the same genes expressed in all inbred lines is consistent with our genomic analysis of CDA genes of maize inbred lines that have the $Z m C D A 1$ and $Z m C D A 2$ gene copy in common (Table 1). The expression analysis also indicates that plants have a CDA gene that is conserved through the monocot-dicot divergence and that the Arabidopsis, rice, and maize gene probably originated from the same ancestral gene. However, we could not find a transcript that matches $Z m C D A 3$. Although it has a complete open reading frame, it is not unexpected that its regulation has changed because of the new position in the genome. One certainly would predict that its expression could not be vital for plant development because its copy is absent in B73. Therefore, it is quite possible that $\mathrm{ZmCDA3}$ is expressed either in very specialized tissue or under specific environmental conditions. Given that this copy arose about 4.5 mya, one would expect that it has maintained its expression potential against deterioration of its sequence. One reason could be that unique haplotypes in the maize genome containing non-allelic gene copies are potentially required for its heterotic properties [10].

\section{Putative functions of CDAs in maize inbred lines}

Function of CDAs is quite diverse, but can be classified by protein structure. The cDNAs of $Z m C D A 1$ and $Z m C D A 2$ described here encode a protein of 184 residues; $Z m C D A 3$ would be 6 residues shorter. Homology search against databases of known protein sequences revealed significant homology $(39.4 \%$ amino acid identity and $74.8 \%$ similarity) with B. subtilis nucleoside deaminase (Fig. 7A). A database search revealed the presence of an active site for nucleoside deaminases, which is conserved within the cytidine deaminase super-family. The cytidine deaminase super-family can be classified into RNA-editing deaminases, cytidine deaminases, nucleoside deaminases and deoxycytidylate deaminases, based on substrate specificity and homology of the active-site sequence [26]. To characterize the putative function of the CDAs in maize, we performed a phylogenetic analysis of CDAs from different organisms with the active site protein sequences (Fig. 7B). The cladogram shows that the active site of maize genes, the rice and the Arabidopsis genes, form a clade, which is close to the bacterial genes encoding nucleotide-modifying enzymes (bootstrap value of 88 ), and constitute the nucleoside deaminase family that is distinct from the mammalian genes encoding RNA or DNA modifying enzymes, cytidine deaminase, and deoxycytidylate deaminase families (Fig. 7C). In general, nucleoside deaminases include adenosine, guanine and cytosine deaminases, which catalyze the de-amination of nucleosides. The functional enzyme is a homodimer. Cytosine deaminase catalyzes the de-amination of cytosine to uracil and ammonia. Because it is found in bacteria and fungi and not in mammals the enzyme is currently of interest for antimicrobial drug design and gene therapy applications against tumours [38-41]. Adenosine deaminases generate inosine at the first position of the anticodon (position 34) of specific tRNAs, which is thought to enlarge the codon recognition capacity during protein synthesis. Guanine deaminases de-aminate guanine to xanthine as part of the utilization of guanine as a nitrogen source. Based on the clustering of ESTs from Arabidopsis, another CDA gene, AtCDA1, has previously been isolated 


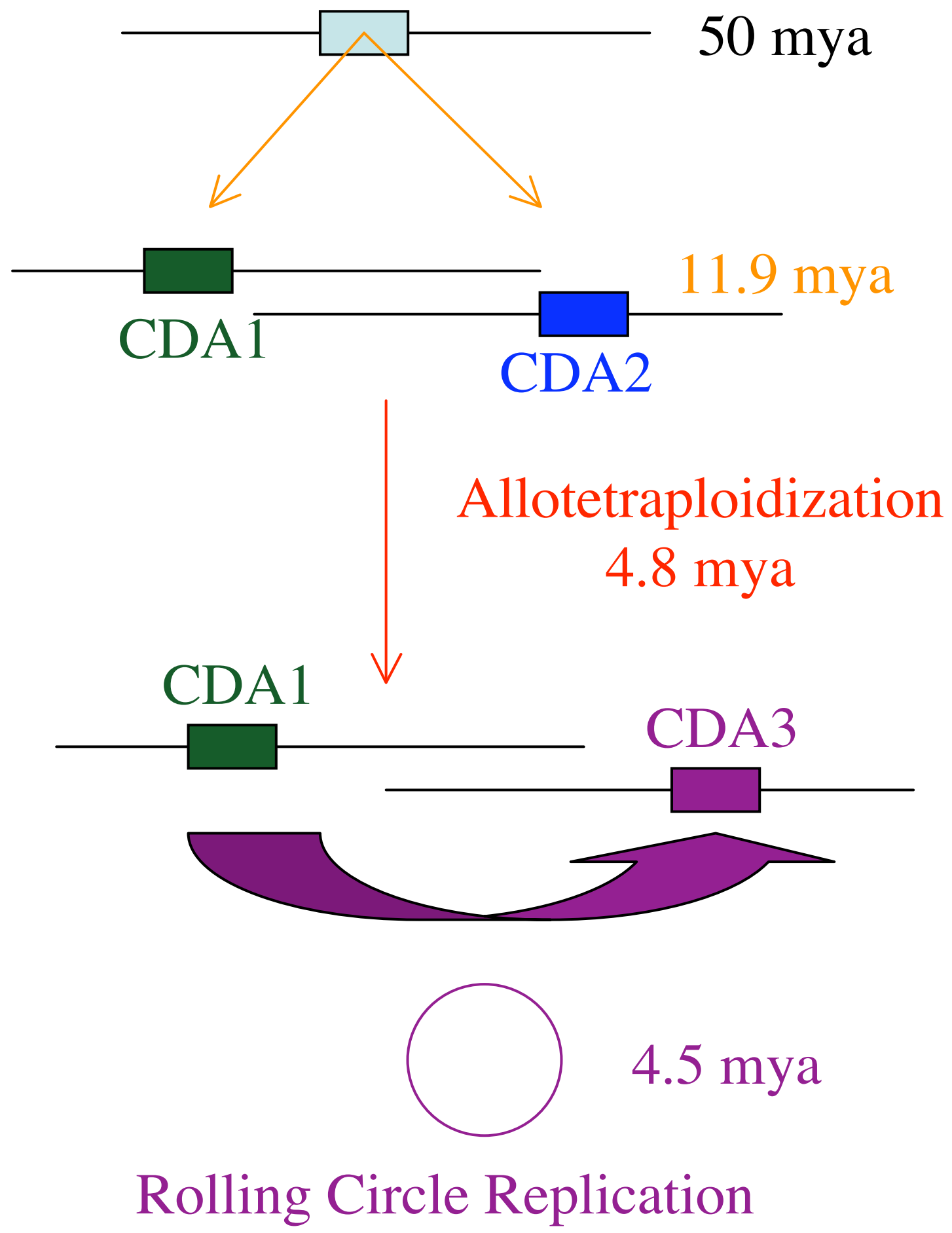

Figure 5

Origin of the $\mathrm{ZmCDA} I, \mathrm{ZmCDA2}$, and $\mathrm{ZmCDA3}$ genes. The progenitors of rice and maize split about 50 mya and the two progenitors of maize about II.9 mya. Each of the progenitors of maize contain a copy of the CDA gene shared with the progenitor of rice (orthologs). When the two progenitors of maize hybridized at about 4.8 mya (allotetraplodization), the maize genome maintained two diverged orthologous CDA gene copies, $Z m C D A I$ and $Z m C D A 2$. In addition, 4.5 mya the $Z m C D A I$ copy on chromosome 7 got copied by the action of a helicase and inserted into chromosome $4 \mathrm{~S}$ close to the $\mathrm{zICI}$ locus, resulting in the $Z m C D A 3$ gene copy, which is therefore a paralogous gene copy. 


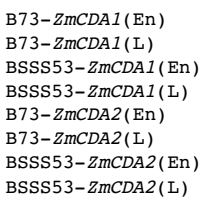

B73-ZMCDA1 (En) B73-ZmCDA1 (L) BSSS53-ZmCDA1 (En) BSSS53-ZMCDA1 (L) B73-ZmCDA2 (En) B73-ZmCDA2 (L) BSSS53-ZmCDA2(En) BSSS53-ZMCDA2 (L)

\section{B73-ZMCDA1 (En)} B73- $Z M C D A 1$ (L) BSSS53-ZmCDA1 (En) BSSS53-ZmCDA1 (L) B73-ZmCDA2 (En) B73-ZmCDA2 (L) BSSS53-ZmCDA2(En) BSSS53-ZMCDA2(L)

B73-ZMCDA1 (En) B73-ZmCDA1 (L) BSSS53-ZmCDA1 (En) BSSS53-ZmCDA1 (L) B73-ZmCDA2 (En) B73-ZmCDA2 (L) BSSS53-ZmCDA2(En) BSSS53-ZMCDA2(L)

B73-ZmCDA1 (En) B73-ZmCDA1 (L) BSSS53-ZmCDA1 (En) BSSS53-ZmCDA1 (L) B73-ZmCDA2 (En) B73-ZmCDA2 (L) BSSS53-ZmCDA2 (En) BSSS53-ZMCDA2 (L)
1 CAACCATGGATGAGGCGCAAGTTGTGGAGTCAAAGGATGGAACCATCTCAGTTGCTTCTGCATTTGTTGCTCATCAGGAAGCTGTACAAGACAGGGATCACAAATTCTTGACAAAAGCAG 1 CAACCATGGATGAGGCGCAACTTTGTGGAGTCAAACCATCGAACCATCTAAT 1 CAACCATGGATGAGGCGCAAGTTGTGGAGTCAAAGGATGGAACCATCTCAGTTGCTTCTGCATTTGCTGCTCATCAGGAAGCTGTGCAAGACAGGGATCACAAATTCTTGACAAAAGCAG 1 CAACCATGGATGAGGCGCAAGTTGTGGAGTCAAAGGATGGAACCATCTCAGTTGCTTCTGCATTTGCTGCTCATCAGGAAGCTGTGCAAGACAGGGATCACAAATTCTTGACAAAAGCAG 1 CAACCATGGATGAGGCGCAAGTTGTGGAGTCAAAGGATGGAACCATCTCAGTTGCTTCTGCATTTGCTGCTCATCAGGAAGCTGTGCAAGACAGGGATCACAAATTCTTGACAAAAGCAG 1 CAACCATGGATGAGGCGCAAGTTGTGGAGTCAAAGGATGGAACCATCTCGGTTGCTTCTGCATTTGCTGGTTATCAGGAAGCTGTGCAAGACAGGGATCACAAATTCTTGACAAAAGCAG

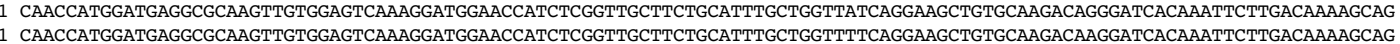
1 CAACCATGGATGAGGCGCAAGT CAACCATGGATGAGGCGCAAGTTGTGGAGTCAAGGA

121 TGGAAGAAGCATACCGAGGAGTCGATTGCGGCGATGGAGGTCCATTTGGAGCAGTTGTCGTCCGTAATGATGAAGTGGTTGTTAGCTGCCATAACATGGTTCTGAAGCACACTGACCCTA 121 TGGAAGAAGCATACCGAGGAGTCGATTGCGGCGATGGAGGTCCATTTGGAGCAGTTGTCGTCCGTAATGATGAAGTGGTTGTTAGCTGCCATAACATGGTTCTGAAGCACACTGACCCTA 121 TGGAAGAAGCATACCGAGGAGTCGATTGCGGTGACGGAGGTCCATTTGGAGCAGTTGTCGTATGTAATGATGAAGTGGTAGTTAGCTGCCATAACATGGTTCTGAAGCACACTGACCCTA 121 TGGAAGAAGCATACCGAGGAGTCGATTGCGGTGACGGAGGTCCATTTGGAGCAGTTGTCGTATGTAATGATGAAGTGGTAGTTAGCTGCCATAACATGGTTCTGAAGCACACTGACCCTA 121 TGGAAGAAGCATATCGAGGAGTCGATTGCGGTGACGGAGGTCCATTCGGAGCAGTTGTCGTCTGTAATGACGAAGTAGTAGTCAGCTGCCATAACATGGTTCTGAAGCACACTGACCCTA 121 TGGAAGAAGCATATCGAGGAGTCGATTGCGGTGACGGAGGTCCATTCGGAGCAGTTGTCGTCTGTAATGACGAAGTAGTAGTCAGCTGCCATAACATGGTTCTGAAGCACACTGACCCTA 121 TGGAAGAAGCATATCGAGGAGTCGATTGCGGTGACGGAGGTCCATTTGGAGCAGTTGTCGTCCGTAATGACGAAGTAGTAGTTAGCTGCCATAACATGGTTCTAAAGCACACTGACCCTA

121 TGGAAGAAGCATATCGAGGAGTCGATTGCGGTGACGGAGGTCCATTTGGAGCAGTTGTCGTCCGTAATGACGAAGTAGTAGTTAGCTGCCATAACATGGTTCTAAAGCACACTGACCCTA

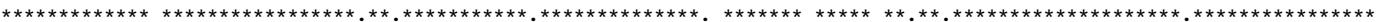

CTGCGCATGCTGAAGTAACTGCAATTAGAGAGGCTTGCAAAAAGCTCGGGAAATCGAGCTCTCTGACTGCGAAATTTACGCGTCCTGCGAGCCATGCCCAATGTGCTTTGGTGCAGTTC 241 CTGCGCATGCTGAAGTAACTGCAATTAGAGAGGCTTGCAAAAAGCTCGGGAAAATCGAGCTCTCTGACTGCGAAATTTACGCGTCCTGCGAGCCATGCCCAATGTGCTTTGGTGCAGTTC 241 CTGCGCATGCTGAAGTAACTGCAATTAGAGAGGCTTGCAAAAAGCTCGGGAAAATCGAGCTCTCTGACTGCGAAATTTACGCGTCCTGCGAGCCATGCCCAATGTGCTTTGGTGCAGTTC 241 CTGCGCATGCTGAAGTAACTGCAATTAGAGAGGCTTGCAAAAAGCTTGGGAAAATTGAGCTCTCAGACTGCGAAATTTACGCGTCCTGCGAGCCATGCCCAATGTGCTTTGGTGCAGTTC 241 CTGCGCATGCTGAAGTAACTGCAATTAGAGAGGCTTGCAAAAAGCTTGGGAAAATTGAGCTCTCAGACTGCGAAATTTACGCGTCCTGCGAGCCATGCCCAATGTGCTTTGGTGCAGTTC 241 CTGCGCATGCTGAAGTAACTGCAATTAGAGAGGCTTGCAAAAAACTTGGGAAAATTGAGCTCTCTGACTGCGAAATTTACGCGTCTTGCGAGCCATGCCCAATGTGCTTTGGTGCAGTTC 241 CTGCGCATGCTGAAGTAACTGCAATTAGAGAGGCTTGCAAAAAACTTGGGAAAATTGAGCTCTCTGACTGCGAAATTTACGCGTCTTGCGAGCCATGCCCAATGTGCTTTGGTGCAGTTC

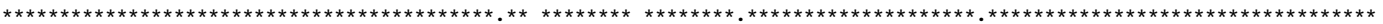

361 ATCTCTCCCGAATCAAGAGGCTGGTTTATGGGGCCAAGGCGGAGGCTGCCATCGCCATTGGGTTTGACGACTTCATTGCAGATGCTCTGAGAGGCACTGGGTTCTACCAGAAGGCCAACA 361 ATCTCTCCCGAATCAAGAGGCTGGTTTATGGGGCCAAGGCGGAGGCTGCCATCGCCATTGGGTTTGACGACTTCATTGCAGATGCTCTGAGAGGCACTGGGTTCTACCAGAAGGCCAAC 361 ATCTCTCCCGGATCAAGAGGCTGGTTTATGGGGCCAAGGCGGAGGCTGCCATCGCCATTGGGTTCGACGACTTCATTGCAGATGCTCTGAGAGGCACTGGGTTCTACCAGAAGGCCAAC 361 ATCTCTCCCGGATCAAGAGGCTGGTTTATGGGGCCAAGGCGGAGGCTGCCATCGCCATTGGGTTCGACGACTTCATTGCAGATGCTCTGAGAGGCACTGGGTTCTACCAGAAGGCCAACA 361 ATCTCTCCCGAATCAAGAGGCTGGTTTATGGGGCCAAGGCAGAGGCTGCCATCGCCATTGGATTTGATGACTTCATTGCAGATGCTCTGAGAGGCACTGGGTTCTACCAGAAGGCCAACT 361 ATCTCTCCCGAATCAAGAGGCTGGTTTATGGGGCCAAGGCAGAGGCTGCCATCGCCATTGGATTTGATGACTTCATTGCAGATGCTCTGAGAGGCACTGGGTTCTACCAGAAGGCCAACT 361 ATCTCTCCCGAATCAAGAGGCTGGTTTATGGGGCCAAGGCAGAGGCTGCCATCGCCATTGGATTTGATGACTTCATTGCAGATGCTCTGAGAGGCACTGGGTTCTACCAGAAGGCCAACT 361 ATCTCTCCCGAATCAAGAGGCTGGTTTATGGGGCAAGGCAGAGGCTGCCATCGCCATTGGATTTGATGACTTCATTGCAGATGCTCTGAGAGGCACTGGGTTCTACCAGAAGGCCAACT ATCTCTCCCGAATCAAGAGGCTGGTTATGGGGCAAGGCAGAGGCTGCCATCGCATTGGATTTGATGACTTCATTGCAGATGCTCTGAGAGGCACTGGGTTCTACCAGAAGGCCAACT

\section{0}

481 TGGAGATCAAGAAGGCCGACGGCAATGGCGCACTGATCGCCGAGCAGGTCTTCGAAAAGACTAAGGAGAAGTTCCAGATGTACTGA 481 TGGAGATCAAGAAGGCCGACGGCAATGGCGCACTGATCGCCGAGCAGGTCTTCGAAAAGACTAAGGAGAAGTTCCAGATGTACTGA 481 TGGAGATCAAGAAGGCCGATGGCAATGGCGCGCTGATTGCCGAGCAGGTCTTCGAAAAGACTAAGGAGAAGTTCCAGATGTACTGA 481 TGGAGATCAAGAAGGCCGATGGCAATGGCGCGCTGATTGCCGAGCAGGTCTTCGAAAAGACTAAGGAGAAGTTCCAGATGTACTGA 481 TGGAGATCAAGAAAGCTGACGGCAATGGTGCATTGATCGCTGAGCAAGTCTTTGAAAAGACTAAAGAGAAGTTCCAGATGTACTGA 481 TGGAGATCAAGAAAGCTGACGGCAATGGTGCATTGATCGCTGAGCAAGTCTTTGAAAAGACTAAAGAGAAGTTCCAGATGTACTGA 481 TGGAGATCAAGAAAGCTGACGGCAATGGTGCATTGATCGCTGAGCAAGTCTTTGAAAAGACTAAAGAGAAGTTCCAGATGTACTGA

481 TGGAGATCAAGAAAGCTGACGGCAATGGTGCATTGATCGCTGAGCAAGTCTTTGAAAAGACTAAAGAGAAGTTCCAGATGTACTGA

\section{6

\section{Figure 6}

Alignment of CDA-related maize cDNAs sequences. Leaf and endosperm tissue samples were used to extract mRNA, which were used to clone CDA-related sequences. They were sequenced and aligned with the genomic coding sequences to determine from which genes they were derived. Alignment was performed using the Laser gene program as described under Methods. Sequences are marked at their beginning.

and its function tested in vitro [30]. The AtCDA1 gene deaminates cytidine, but cannot use RNA as a substrate. Therefore, it resembles the enzyme found in E. coli with the same function and differs in its structure from the mammalian proteins. Nevertheless, we know that RNA editing occurs in plant organelles [42]. Moreover, biochemical assays have shown that extracts contain an enzyme activity consistent with the RNA-editing function [43]. Because most proteins in chloroplast are derived from the nuclear genome, one would also expect CDA encoding genes in the plant genome that are closer to the mammalian clade of CDAs capable of modifying RNA or even DNA. As the Southern blot of genomic DNA from different inbred lines indicates the maize genome contains other CDA-related gene sequences that could account for these other functions (Fig. 2).

\section{Conclusion}

A new Helitron has been discovered whose internal sequences contain an intact coding sequence for a cytidine deaminase. Because previous Helitron sequences were missing intact coding sequences it was not possible to determine when they were copied from their donor site. From sequence alignments with the two orthologous copies of the maize genome and the copy of the rice genome, it appears that the Helitron-based movement of the paralogous CDA gene copy occurred about 4.5 mya. Conservation of the coding regions including intronic sequences of $94.6 \%$ between the orthologous and the paralogous gene copies suggests that the paralogous gene copy is required although not as an essential gene. Therefore, it is likely to be conditionally induced and/or provides a quantitative trait as a selective advantage rather than a typical house- 


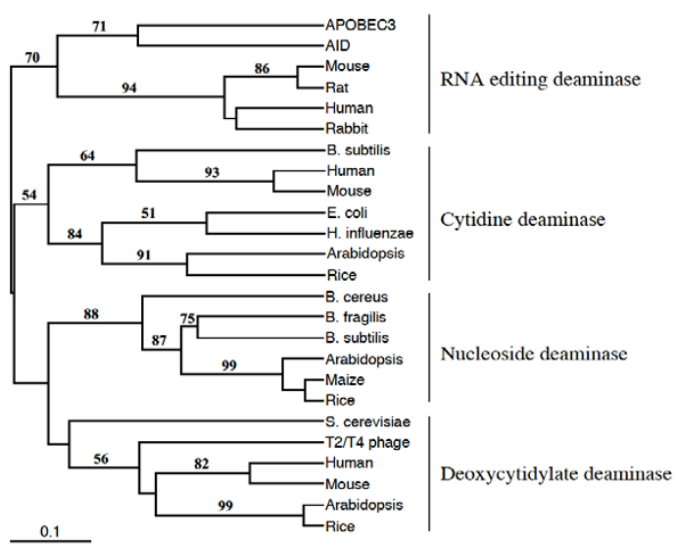

\section{Figure 7}

CDA-related genes according to their function. A) Alignment of amino acid sequences of maize cytidine deaminase and B. subtilis nucleoside deaminase. Identical and conserved amino acids are marked by asterisks and dots, respectively; hyphens represent gaps. B) Alignment of the active sites of cytidine deaminases. Accession numbers of amino acid sequences used here are as follows: [GenBank: APOBEC3, Q9HCl6; AID, Q9GZX7]; mouse [GenBank: APOBECI, U2195I]; rat [GenBank: APOBECI, L07I I4]; human [GenBank: APOBECI, L25877]; rabbit [GenBank: APOBECI, U10695]; B. subtilis cytidine deaminase [GenBank: PI 9079]; human cytidine deaminase [GenBank: L27943]; mouse cytidine deaminase [GenBank: P56389]; E. coli cytidine deaminase [GenBank: PI3652]; H. influenzae cytidine deaminase [GenBank: U328I4]; Arabidopsis cytidine deaminase [GenBank: AJ005687]; rice cytidine deaminase [GenBank: NP 916608]; B. cereus nucleoside deaminase [GenBank: NP 829925]; B. cereus nucleoside deaminase [GenBank: AAP07|26]; B. fragilis nucleoside deaminase [GenBank: BAD48383]; $B$. subtilis nucleoside deaminase [GenBank: O34598]; Arabidopsis nucleoside deaminase [GenBank: NP 198157]; maize nucleoside deaminase, this work [GenBank: EF 106973]; rice nucleoside deaminase [GenBank: XP 470319]; S. cerevisiae deoxycytidylate deaminase [GenBank: P06773]; T2/T4 phage deoxycytidylate deaminase [GenBank: P008I4]; human deoxycytidylate deaminase [GenBank: LI2136]; mouse deoxycytidylate deaminase [GenBank: O8K2D6]; Arabidopsis deoxycytidylate deaminase [GenBank: ABE66000]; rice deoxycytidylate deaminase [GenBank: BAD87/46]. C) The phylogenetic tree was constructed based on the active site of cytidine deaminases using the PAUP 4.0b I0 UPGMA method [50]. Numbers indicate bootstrap values (above $50 \%$ with 1000 replications). 
keeping function. One can envision that it is expressed under certain environmental conditions. Another possibility is that it is under the control of a regulatory factor that itself is encoded by a paralogous gene sequence and is only present in unique haplotypes. In such a scenario, one would have to combine these two haplotypes by crossing to enable the expression of the paralogous CDA gene copy. While combinatorial regulatory circuits for a CDA function still remains to be explored, it can serve as a paradigm of how one could achieve unique phenotypes that require the right inbred strains to form a hybrid.

\section{Methods}

\section{Plant materials}

Our laboratory stocks of maize inbred lines A632, A636, A654, B37, Mo17, W22, W23, W64A, CM37, T232, CO159, Tx303, A188, B73, and BSSS53 were obtained from the NPG collection [44].

\section{Identification of B73 clone b0390II 0}

The CDA gene sequence linked to the z1C1 locus on chromosome $4 \mathrm{~S}$ of BSSS53 was subjected to a BLAST search [31] of the B73 BESs on our local server. One BAC end sequence [GenBank: CG435284] had homology to the coding region of a CDA gene. We searched the $\mathrm{B} 73$ maize physical map for a BAC contig [45] containing the positive clone. Contig \#299 on chromosome 7 was identified. However, after positioning the CDA containing BES within the FPC BAC clone b0390I10 was selected for sequencing to obtain all CDA flanking sequences [GenBank: EF106973].

\section{Southern blot hybridiztion}

A total of $25 \mu \mathrm{g}$ of maize genomic DNA was digested with different restriction enzymes. DNA fragments were separated on $1.0 \%$ agarose gels, blotted on Hybond-XL nylon membranes (Amersham Biosciences), and the membranes were hybridized with CDA CDNA sequences as a probe that had been labeled as described previously [46].

\section{Genomic PCR and Reverse-transcription PCR (RT-PCR)}

$\mathrm{CDA}$ gene sequences were amplified from total genomic DNA of 15 inbred lines by using primer pairs, covering exon 2, intron 2, exon 3, intron 3, and exon 4:

\section{ZmCDAF3, CATCTGGAACTTCTCCTTAG,}

\section{ZmCDAR3, ACAGGGATCACAAATTCTTG;}

the last exon, exon 4, of the CDA gene was also amplified with primer pairs:

ZmCDAF4, GACTTCATTGCAGATGCTCTG，
Total RNA of mature leaves and immature endosperm 20 days after pollination of maize inbred lines was extracted with the RNeasy Plant Mini Kit (Qiagen). We reverse-transcribed RNA to cDNA using SuperScript First-Strand Synthesis System (Invitrogen) with an oligo(dT) primer.

Amplification was then carried out for the $Z m C D A 1$ and $\mathrm{ZmCDA2}$ genes with primer pairs:

\section{ZmCDAF1, CAACCATGGATGAGGCGCAAG,}

ZmCDAR1, TCAGTACATCTGGAACTTCTC, and for the $Z m C D A 3$ gene with primer pairs:

\section{ZmCDAF2, ATGGAGTCAAAGGATGGAAC,}

\section{ZmCDAR1, TCAGTACATCTGGAACTTCTC.}

\section{DNA and CDNA sequencing and analysis}

The BAC clone was sequenced by DNA shotgun sequencing with an Applied Biosystems $3730 \times 1$ DNA Analyzer using universal primers [47]. PCR and RT-PCR products were cloned and also sequenced with universal primers [GenBank: EF105328-EF105335]. DNA sequences were analyzed with the Laser gene application kit (DNAstar, Madison, WI). Multiple sequence alignments were carried out by the program ClustalW [34].

\section{Estimation of the rate of synonymous substitution and divergence time}

We used CDA gene exon sequences to estimate synonymous substitution rates $(\mathrm{k})$ using standard methods [48]. The divergence time $\mathrm{T}$ between maize, sorghum, and rice was set at 50 million years [36]. The substitution rate $\mathrm{k}$ was calculated according to the formula $\mathrm{k}=$ $[\mathrm{Ks}(\mathrm{a})+\mathrm{Ks}(\mathrm{b})] / 2 \mathrm{2} 2 \mathrm{~T}$, where $\mathrm{Ks}(\mathrm{a})$ is the relative synonymous substitution rate between maize and rice, and $\mathrm{Ks}(\mathrm{b})$ the relative synonymous substitution rate between sorghum and rice.

For estimation of the divergence time t of two sequences, we used $\mathrm{t}=\mathrm{ds} / 2 \mathrm{k}$.

\section{Authors' contributions}

$\mathrm{J}-\mathrm{H}$. X. performed the experiments and J. M. helped with the design of the experiments and the writing of the manuscript. The final version of the manuscript has been read and approved by all authors.

\section{Acknowledgements}

This work has been supported by grants of the Department of Energy (DOE) \#DE-FG05-95ER20194 and the National Science Foundation (NSF) DBI 03-20683. 


\section{References}

I. Gale MD, Devos KM: Comparative genetics in the grasses. Proc Natl Acad Sci U S A 1998, 95(5): |97|-1974.

2. Ilic K, SanMiguel PJ, Bennetzen JL: A complex history of rearrangement in an orthologous region of the maize, sorghum, and rice genomes. Proc Natl Acad Sci U S A 2003, I 00(2 I): | 2265- I 2270.

3. Song R, Llaca V, Messing J: Mosaic organization of orthologous sequences in grass genomes. Genome Res 2002, I 2(10): I549-1555.

4. Lai J, Ma J, Swigonova Z, Ramakrishna W, Linton E, Llaca V, Tanyolac B, Park YJ, Jeong OY, Bennetzen JL, Messing J: Gene loss and movement in the maize genome. Genome Res 2004 I4(IOA): |924-1931.

5. Bruggmann R, Bharti AK, Gundlach H, Lai J, Young S, Pontaroli AC, Wei F, Haberer G, Fuks G, Du C, Raymond C, Estep MC, Liu R, Bennetzen JL, Chan AP, Rabinowicz PD, Quackenbush J, Barbazuk WB, Wing RA, Birren B, Nusbaum C, Rounsley S, Mayer KF, Messing J: Uneven chromosome contraction and expansion in the maize genome. Genome Res 2006, I 6(I 0): | 24|-|25|

6. Thuriaux $P$ : Is recombination confined to structural genes on the eukaryotic genome? Nature 1977, 268(56 I 9):460-462.

7. Dooner HK, Martinez-Ferez IM: Recombination occurs uniformly within the bronze gene, a meiotic recombination hotspot in the maize genome. Plant Cell 1997, 9(9): |633- I646.

8. Timmermans MC, Das OP, Messing J: Characterization of a meiotic crossover in maize identified by a restriction fragment length polymorphism-based method. Genetics 1996 |43(4): |77|-|783.

9. $\mathrm{Fu} \mathrm{H}$, Dooner HK: Intraspecific violation of genetic colinearity and its implications in maize. Proc Natl Acad Sci U S A 2002, 99( I 4):9573-9578.

10. Song R, Messing J: Gene expression of a gene family in maize based on noncollinear haplotypes. Proc Natl Acad Sci U S A 2003, I 00( I 5):9055-9060.

I I. Brunner S, Fengler K, Morgante M, Tingey S, Rafalski A: Evolution of DNA Sequence Nonhomologies among Maize Inbreds. Plant Cell 2005, I 7(2):343-360.

12. Song R, Llaca V, Linton E, Messing J: Sequence, regulation, and evolution of the maize 22-kD alpha zein gene family. Genome Res 200I, II (II):1817-1825.

13. Jiang N, Bao Z, Zhang X, Eddy SR, Wessler SR: Pack-MULE transposable elements mediate gene evolution in plants. Nature 2004, 43 I (7008):569-573.

14. Ma J, Bennetzen JL: Rapid recent growth and divergence of rice nuclear genomes. Proc Natl Acad Sci U S A 2004 I 0 I(34): I 2404- I 24 I0.

15. Han B, Xue Y: Genome-wide intraspecific DNA-sequence variations in rice. Curr Opin Plant Biol 2003, 6(2): I34-138.

16. Scherrer B, Isidore E, Klein P, Kim JS, Bellec A, Chalhoub B, Keller B Feuillet $C$ : Large intraspecific haplotype variability at the Rph7 locus results from rapid and recent divergence in the barley genome. Plant Cell 2005, I 7(2):36I-374.

17. Rodriguez-Trelles F, Tarrio R, Ayala F]: Convergent neofunctionalization by positive Darwinian selection after ancient recurrent duplications of the xanthine dehydrogenase gene. Proc Natl Acad Sci U S A 2003, I 00(23): I34|3-134|7.

18. Ono R, Nakamura K, Inoue K, Naruse M, Usami T, Wakisaka-Saito N, Hino T, Suzuki-Migishima R, Ogonuki N, Miki H, Kohda T, Ogura A, Yokoyama M, Kaneko-Ishino T, Ishino F: Deletion of Peg I0, an imprinted gene acquired from a retrotransposon, causes early embryonic lethality. Nat Genet 2006, 38(I): I0I-106.

19. Danilevskaya ON, Hermon P, Hantke S, Muszynski MG, Kollipara K, Ananiev EV: Duplicated fie genes in maize: expression pattern and imprinting suggest distinct functions. Plant Cell 2003, I 5(2):425-438.

20. Du C, Swigonova Z, Messing J: Retrotranspositions in orthologous regions of closely related species. BMC Evol Biol 2006, 6(I):62.

21. Morgante M, Brunner S, Pea G, Fengler K, Zuccolo A, Rafalski A: Gene duplication and exon shuffling by helitron-like transposons generate intraspecies diversity in maize. Nat Genet 2005, 37(9):997-1002.

22. Lai J, Li Y, Messing J, Dooner HK: Gene movement by Helitron transposons contributes to the haplotype variability of maize. Proc Natl Acad Sci U S A 2005, I 02(25):9068-9073.
23. Kapitonov VV, Jurka J: Rolling-circle transposons in eukaryotes. Proc Natl Acad Sci U S A 200I, 98( I 5):87| 4-87I9.

24. Lal SK, Giroux MJ, Brendel V, Vallejos CE, Hannah LC: The maize genome contains a helitron insertion. Plant Cell 2003, I5(2):38|-39|

25. Gupta S, Gallavotti A, Stryker GA, Schmidt RJ, Lal SK: A novel class of Helitron-related transposable elements in maize contain portions of multiple pseudogenes. Plant Mol Biol 2005, 57(I): II5-I27.

26. Navaratnam N, Bhattacharya S, Fujino T, Patel D, Jarmuz AL, Scott J: Evolutionary origins of apoB mRNA editing: catalysis by a cytidine deaminase that has acquired a novel RNA-binding motif at its active site. Cell 1995, 8I(2): 187-195.

27. Doi T, Kinoshita K, Ikegawa M, Muramatsu M, Honjo T: De novo protein synthesis is required for the activation-induced cytidine deaminase function in class-switch recombination. Proc Natl Acad Sci U S A 2003, I 00(5):2634-2638.

28. Petersen-Mahrt SK, Harris RS, Neuberger MS: AID mutates E. coli suggesting a DNA deamination mechanism for antibody diversification. Nature 2002, 4 I 8(6893):99-I03.

29. Rada C, Williams GT, Nilsen H, Barnes DE, Lindahl T, Neuberger MS: Immunoglobulin isotype switching is inhibited and somatic hypermutation perturbed in UNG-deficient mice. Curr Biol 2002, I 2(20): I748-1755.

30. Faivre-Nitschke SE, Grienenberger JM, Gualberto JM: A prokaryotic-type cytidine deaminase from Arabidopsis thaliana gene expression and functional characterization. Eur J Biochem I999, 263(3):896-903.

31. Altschul SF, Madden TL, Schaffer AA, Zhang J, Zhang Z, Miller W, Lipman DJ: Gapped BLAST and PSI-BLAST: a new generation of protein database search programs. Nucleic Acids Res 1997, 25( I 7):3389-3402.

32. Messing J, Bharti AK, Karlowski WM, Gundlach H, Kim HR, Yu Y, Wei F, Fuks G, Soderlund CA, Mayer KF, Wing RA: Sequence composition and genome organization of maize. Proc Natl Acad Sci U S A 2004, I 0 I (40): | 4349-|4354.

33. Nelson WM, Bharti AK, Butler E, Wei F, Fuks G, Kim HR, Wing RA Messing J, Soderlund C: Whole-Genome Validation of HighInformation-Content Fingerprinting. Plant Physiol 2005, I39(I):27-38

34. Thompson JD, Gibson TJ, Plewniak F, Jeanmougin F, Higgins DG: The CLUSTAL $X$ windows interface: flexible strategies for multiple sequence alignment aided by quality analysis tools. Nucleic Acids Res 1997, 25(24):4876-4882.

35. Wilson WA, Harrington SE, Woodman WL, Lee M, Sorrells ME, McCouch SR: Inferences on the genome structure of progenitor maize through comparative analysis of rice, maize and the domesticated panicoids. Genetics 1999, I 53(I):453-473.

36. Wolfe KH, Gouy M, Yang YW, Sharp PM, Li WH: Date of the monocot-dicot divergence estimated from chloroplast DNA sequence data. Proc Natl Acad Sci U S A 1989, 86( I 6):620I-6205.

37. Swigonova Z, Lai J, Ma J, Ramakrishna W, Llaca V, Bennetzen JL, Messing J: Close split of sorghum and maize genome progenitors. Genome Res 2004, I4(I 0A): 1916-1923.

38. Mullen CA, Kilstrup M, Blaese RM: Transfer of the bacterial gene for cytosine deaminase to mammalian cells confers lethal sensitivity to 5 -fluorocytosine: a negative selection system. Proc Natl Acad Sci U S A 1992, 89(1):33-37.

39. Ohwada A, Hirschowitz EA, Crystal RG: Regional delivery of an adenovirus vector containing the Escherichia coli cytosine deaminase gene to provide local activation of 5 -fluorocytosine to suppress the growth of colon carcinoma metastatic to liver. Hum Gene Ther 1996, 7(13): I567-I576.

40. Freytag SO, Khil M, Stricker H, Peabody J, Menon M, DePeralta-Venturina M, Nafziger D, Pegg J, Paielli D, Brown S, Barton K, Lu M, Aguilar-Cordova E, Kim JH: Phase I study of replication-competent adenovirus-mediated double suicide gene therapy for the treatment of locally recurrent prostate cancer. Cancer Res 2002, 62(I 7):4968-4976.

4I. Mahan SD, Ireton GC, Knoeber C, Stoddard BL, Black ME: Random mutagenesis and selection of Escherichia coli cytosine deaminase for cancer gene therapy. Protein Eng Des Sel 2004, I 7(8):625-633.

42. Bock R, Kossel H, Maliga P: Introduction of a heterologous editing site into the tobacco plastid genome: the lack of RNA 
editing leads to a mutant phenotype. Embo J 1994, 13(19):4623-4628.

43. Hegeman CE, Hayes ML, Hanson MR: Substrate and cofactor requirements for RNA editing of chloroplast transcripts in Arabidopsis in vitro. Plant J 2005, 42(I): I24-132.

44. National-Plant-Germplasm-collection: www.ars-grin.gov/npgs.

45. WebFPC: http://www.genome.arizona.edu/fpc/WebAGCoL/ maize/WebFPC/.

46. Song R, Messing J: Contiguous genomic DNA sequence comprising the 19-kD zein gene family from maize. Plant Physiol 2002, 130(4):1626-1635.

47. Vieira J, Messing J: The pUC plasmids, an MI3mp7-derived system for insertion mutagenesis and sequencing with synthetic universal primers. Gene 1982, 19(3):259-268.

48. Nei M, Gojobori T: Simple methods for estimating the numbers of synonymous and nonsynonymous nucleotide substitutions. Mol Biol Evol 1986, 3(5):418-426.

49. Gallavotti A, Zhao Q, Kyozuka J, Meeley RB, Ritter MK, Doebley JF, $\mathrm{Pe} M E$, Schmidt RJ: The role of barren stalkl in the architecture of maize. Nature 2004, 432(70I7):630-635.

50. PAUP: http://paup.csit.fsu.edul.

Publish with Biomed Central and every scientist can read your work free of charge

"BioMed Central will be the most significant development for disseminating the results of biomedical research in our lifetime. "

Sir Paul Nurse, Cancer Research UK

Your research papers will be:

- available free of charge to the entire biomedical community

- peer reviewed and published immediately upon acceptance

- cited in PubMed and archived on PubMed Central

- yours - you keep the copyright 